\title{
Teleoperation Control of a Redundant Continuum Manipulator Using a Non-Redundant Rigid-Link Master
}

\author{
Apoorva D. Kapadia ${ }^{\dagger}$, Ian. D. Walker and Enver Tatlicioglu
}

\begin{abstract}
In this paper, teleoperated control of a kinematically redundant, continuum slave manipulator with a nonredundant, rigid-link master system is considered. This problem is novel because the self-motion of the redundant robot can be utilized to achieve secondary control objectives while allowing the user to concentrate on controlling only the tip of the slave system. To that end, feedback linearizing controllers are proposed for both the master and slave systems, whose effectiveness is demonstrated using numerical simulations for the case of singularity avoidance as a subtask.
\end{abstract}

\section{INTRODUCTION}

Despite the numerous possible applications of continuum arms and the extensive work in teleoperation of robot manipulators, it is perhaps surprising that teleoperation of continuum arms provides a novel consideration. A teleoperation system enables a user to execute a task using an output system (i.e. a slave) while manipulating an input system (i.e. a master) in a remote environment. Teleoperation of robotic systems has been invaluable for numerous applications such as handling unstructured or hazardous materials, maneuvering underwater vehicles, search and rescue, and most recently in assisting medical procedures [1], [2].

Continuum robots are loosely defined as manipulators with continuous backbones, whose modeling and control is an emerging area in the field of robotics [3], [4], [5], [6], [7], [8], [9], [10]. These manipulators draw their design inspirations from the Animals Kingdom such as octopus arms, squid tentacles, and elephant trunks, and detailed extensively in [11]. Manipulators with continuum backbones are able to bend at any point along their structure, and thus have the potential to perform operations not feasible with conventional robots, such as whole-arm grasping, manipulations of objects having arbitrary and a priori unknown shapes, and navigation in unstructured environments especially in search and rescue situations. Kinematics for continuum manipulators have been extensively developed, a review of which can be found in [12] while research in dynamics is active [13], [14], [15], [16], [17].

$\dagger$ To whom all correspondence should be addressed.

A.D. Kapadia and I.D. Walker are with the Dept. of Electrical \& Computer Engineering, Clemson University, Clemson, SC-29634-0915 ((akapadi, iwalker)@clemson.edu).

E. Tatlicioglu is with the Department of Electrical \& Electronics Engineering, Izmir Institute of Technology, Urla, Izmir, 35430 Turkey (enver@envertatlicioglu.com).

This research was supported by the U.S. NSF under grants IIS-0844954 and IIS-0904116
As with traditional rigid-link kinematically redundant robots, due to the excess of degrees of freedom (DOFs) of many continuum manipulators in comparison to the taskspace in which they work, motion in the null space of the Jacobian exists with no bearing on the position and orientation of the tip, and is commonly referred to as self motion. This is useful for various sub-tasks such as singularity or obstacle avoidance. However, the use of continuum manipulators often complicates the control design, due to the dearth of mathematical models for null-space motion and subsequently control strategies. Configuration-space controllers for contin-

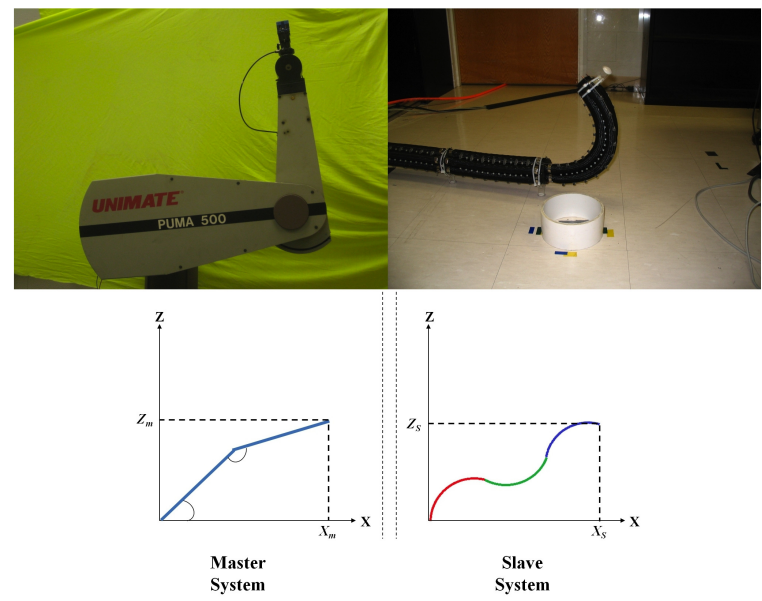

Fig. 1. Example of a non-redundant, rigid-link master controlling a redundant, continuum slave system and a general representation of the proposed teleoperation system

uum manipulators have been established [18], [19] [20], [21] while Kapadia and Walker recently developed a model-based task-space controller for the Octarm continuum manipulator [22] which could regulate any location on the manipulator backbone. Teleoperation of continuum robot arms using a 3DOF video-gaming joystick was initially discussed in [23], though in this case the master system was not a robotic manipulator itself.

Due to the relative complexity of the physical design and construction of continuum arms as compared to more conventional robots, teleoperation of such robots have the disadvantage of not always having a kinematically similar system to serve as a master, as shown in Figure 1. In such instances, and where only a non-redundant system might be available to serve as a master, a solution might be found in the task-space of both systems where an additional control element could be designed to achieve secondary objectives such as obstacle or singularity avoidance with no user input 
necessary to that end. Thus, the master system would be relieved of any duties beyond controlling the end-effector trajectory of the non-redundant master.

Related literature focuses on a similar problem ofthe master and slave systems having dissimilar kinematics, primarily dealing with rigid-link structures, and not continuum arms, as focused on in this article. Kim et al. [24] classified the dissimilar kinematics teleoperation problem by comparing the DOFs of master and slave systems. Herndon et al. [25] were the first to use kinematically redundant robot manipulators in a dual-arm teleoperator system to avoid obstacles in space applications. Nguyen et al. [26] developed an adaptive joint-space controller for a dual arm system where both slave arms were kinematically redundant robot manipulators; however, kinematic redundancy was not considered in the control development. Jansen et al. [27] designed a stiffness controller for a teleoperator system with a 7 DOF slave and a 6 DOF master, utilized extended task-space techniques for redundancy resolution and Euler parameters to avoid artificial singularities. Those results were extended in [28], by adding passivity to the overall system. Hwang et al. [29] outlined the performance of a teleoperator system with a kinematically redundant slave system, but did not provide robustness when the robot operated close to its kinematic singularities or for high joint velocities. Nanayakkara et al. [30] utilized neural networks to compensate for the uncertain master system and environmental dynamics. Goel et al. [31] demonstrated experimental results where a planar $3 \mathrm{DOF}$ robot manipulator was considered as the slave system. Kinematic control was utilized and the kinematic redundancy was used to maintain control even with joint failures. Buss et al. [32], [33], [34], [35] considered experimental verification of haptic teleoperation with a kinematically redundant slave system. In [36], Hayakawa et al. used a 7 DOF manipulator for the slave system and the master system had 6 DOF. The redundancy in the slave system was utilized to move the robot away from its singular configurations but only with the help of the user. Gosselin et al. [37] demonstrated experimental results with a kinematically redundant haptic device however did not make use of the redundancy resolution. Recently, Nath et al. [38] discussed teleoperation with kinematically redundant master and slave systems and utilized the redundancy in both systems to achieve secondary objectives where the master and slave systems were kinematically similar. Most of the aforementioned works focused on experimental verification [36] - [38] while others such as [26], [29], [37], and [39] did not take advantage of all the properties of kinematically redundant manipulators, especially for control design. Thus, a control framework is required for teleoperation with dissimilar kinematics, particularly for a kinematically redundant continuum arm as the slave system.

This paper focuses on the teleoperation control of a continuum manipulator slave, specifically the Octarm [9], using a non-redundant, revolute, rigid-link robot as the master, and shown schematically in Figure 1. Due to the kinematic redundancy in the slave system, explicit control of the shape of the continuum manipulator by the user via the non-redundant master system is not possible. It is the objective of this work to develop a controller to make use of the redundancy resolution by automatically satisfying secondary objectives while the user focuses on control of the slave system's tip thus reducing the user workload. In the subsequent control development, dynamic and kinematic models and the interaction forces acting on both master and slave systems are assumed to be measurable. Based on the exact model knowledge, feedback linearizing controllers are designed for both master and slave systems.

The designed controllers ensure that the desired trajectory tracking errors for both the master and slave systems are driven to zero exponentially fast. Additionally the slave system controller allows the integration of a null-space controller to make use of the redundancy resolution via the design of a null-space velocity tracking error [40]. The null-space velocity tracking error is shown to go to zero monotonically. To that end, the general sub-task controller developed in [41] is extended to integrate secondary control objectives.

\section{Mathematical Model}

\section{A. Dynamic Model}

The dynamic models for both the master and slave systems are described by the standard Euler-Lagrangian form

$$
M_{i} \ddot{q}_{i}+N_{i}=T_{i}+J_{i}^{T} F_{i}
$$

where $i=1$ represents the master system and $i=2$ represents the slave system. The number of joints of the master system is denoted by $n_{1}$, whereas $n_{2}$ represents the number of slave system section lengths and curvatures.

The number of degrees of freedom of master and slave systems are equal to $n_{1} \in \mathbb{N}$ and $n_{2} \in \mathbb{N}$ respectively, where $n_{2}>n_{1}$, highlighting the kinematic dissimilarity between the two systems. In $(1), q_{1}(t), \dot{q}_{1}(t), \ddot{q}_{1}(t) \in \mathbb{R}^{n_{1}}$ denote the position, velocity and acceleration of the master system rigid-links, while, $q_{2}(t), \dot{q}_{2}(t), \ddot{q}_{2}(t) \in \mathbb{R}^{n_{2}}$ represents the slave system section lengths and curvatures, and their firstand second-order rates of changes respectively. $M_{i}\left(q_{i}\right) \in$ $\mathbb{R}^{n_{i} \times n_{i}}$ represents the inertia matrix, $N_{i}\left(q_{i}, \dot{q}_{i}\right) \in \mathbb{R}^{n_{i}}$ represents other dynamic effects (centripetal-Coriolis effects, gravitational forces, and other dynamic frictional effects), $T_{i}(t) \in \mathbb{R}^{n_{i}}$ represents the control input vector, $F_{i}(t) \in \mathbb{R}^{n_{i}}$ represents the task-space interaction forces, and $J_{i}\left(q_{i}\right) \in$ $\mathbb{R}^{n_{1} \times n_{i}}$ represents the Jacobian matrices for the master and slave systems. The inertia matrix $M_{i}(\cdot)$ is symmetric and positive-definite, and satisfies the following inequalities [42]

$$
m_{1 i}\|\xi\|^{2} \leq \xi^{T} M_{i}(\cdot) \xi \leq m_{2 i}\|\xi\|^{2} \quad \forall \xi \in \mathbb{R}^{n_{i}}
$$

where $m_{1 i}, m_{2 i} \in \mathbb{R}$ are positive constants and $\|\cdot\|$ denotes the standard Euclidean norm.

\section{B. Kinematic Model}

The kinematic models for the master and slave systems are defined as

$$
x_{i} \triangleq f\left(q_{i}\right)
$$


where $x_{i}(t) \in \mathbb{R}^{n_{1}}$ is the task-space position and $f\left(q_{i}\right) \in$ $\mathbb{R}^{n_{i}}$ represents the forward kinematics of the manipulator.The first and second time derivatives of (3) are found to be

$$
\begin{gathered}
\dot{x}_{i}=J_{i} \dot{q}_{i} \\
\ddot{x}_{i}=\dot{J}_{i} \dot{q}_{i}+J_{i} \ddot{q}_{i} .
\end{gathered}
$$

Rearranging the terms in (4) results in

$$
\begin{aligned}
& \ddot{q}_{1}=J_{1}^{-1}\left(\ddot{x}_{1}-\dot{J}_{1} \dot{q}_{1}\right) \\
& \ddot{q}_{2}=J_{2}^{+}\left(\ddot{x}_{2}-\dot{J}_{2} \dot{q}_{2}\right)+\ddot{q}_{N}
\end{aligned}
$$

where $\ddot{q}_{N}(t) \in \mathbb{R}^{n_{2}}$ is an auxiliary vector in the null-space of $J_{2}(\cdot)$ and $J_{2}^{+}\left(q_{2}\right) \in \mathbb{R}^{n_{2} \times n_{1}}$ is the Moore-Penrose pseudoinverse of the Jacobian of the slave system defined as

$$
J_{2}^{+} \triangleq J_{2}^{T}\left(J_{2} J_{2}^{T}\right)^{-1} \text {. }
$$

From (7), it can be seen that ${ }^{1}$

$$
J_{2} J_{2}^{+}=I_{n_{1}} .
$$

Also, the matrix $\left(I_{n_{2}}-J_{2}^{+} J_{2}\right)$ satisfies the following Moore-Penrose properties

$$
\begin{aligned}
\left(I_{n_{2}}-J_{2}^{+} J_{2}\right)\left(I_{n_{2}}-J_{2}^{+} J_{2}\right) & =I_{n_{2}}-J_{2}^{+} J_{2} \\
\left(I_{n_{2}}-J_{2}^{+} J_{2}\right) J_{2}^{+} & =0_{n_{2} \times n_{1}} .
\end{aligned}
$$

Assumption 1: The kinematic and dynamic terms for a general revolute robot manipulator used as the master system, and the Octarm used as the slave system $\left(M_{i}\left(q_{i}\right), N_{i}\left(q_{i}, \dot{q}_{i}\right)\right.$, $J_{i}\left(q_{i}\right)$ and $\left.J_{2}^{+}\left(q_{2}\right)\right)$ are dependent on $q_{i}(t)$ only as arguments of sinusoidal trigonometric functions, and hence, remain bounded for all possible $q_{i}(t)$. Thus it is assumed that if $x_{i}(t) \in \mathcal{L}_{\infty}$ then $q_{i}(t) \in \mathcal{L}_{\infty}$.

\section{The Task Space}

The master system is represented by a 2-link, planar, revolute robot manipulator, thus $q_{1}(t)$ refers to the manipulator joint angles and $n_{1}=2$ thus also defining the planar taskspace as $x=\left[\begin{array}{ll}X & Z\end{array}\right]^{T}$, where $X(t) \in \mathbb{R}$ and $Z(t) \in \mathbb{R}$ are the scalar Euclidean coordinates of the master system end-effector.

Since the slave system is a continuum manipulator, $q_{2}(t) \in$ $\mathbb{R}^{n_{2}}$ represents the manipulator section lengths and curvatures. For the 3-section Octarm used in the plane of motion of the master, to ensure redundancy, $q_{2}(t) \in \mathbb{R}^{6}$ and $q_{2}(t)=\left[d_{1}, d_{2}, d_{3}, \kappa_{1}, \kappa_{2}, \kappa_{3}\right]^{T}$, are the extension lengths and curvatures for each of the three sections respectively.

\section{TASK-SPACE CONTROLler DEVElopment}

The primary design objective is to formulate a control input that ensures that the tip of the slave system tracks the end-effector of the master system which in turn tracks a desired task-space trajectory. The subsequent development is based on the assumption that the configuration-space positions and velocities are measurable for both master and

\footnotetext{
${ }^{1}$ Throughout the paper, $I_{n}$ and $0_{m \times r}$ will be used to represent an $n \times n$ standard identity matrix and an $m \times r$ zero matrix, respectively.
}

slave systems. The task-space tracking error for the master system denoted by $e_{1}(t) \in \mathbb{R}^{n_{1}}$ is defined as follows

$$
e_{1} \triangleq x_{d}-x_{1}
$$

where $x_{d}(t) \in \mathbb{R}^{n_{1}}$ is the desired task-space trajectory to be tracked by the master system. The desired trajectory is chosen such that $x_{d}(t), \dot{x}_{d}(t)$, and $\ddot{x}_{d}(t) \in \mathcal{L}_{\infty}$. Based on exact model knowledge, the following feedback linearizing controller is designed for the master system

$$
\begin{gathered}
T_{1} \triangleq M_{1} u_{1}+N_{1}-J_{1}^{T} F_{1}, \\
u_{1} \triangleq J_{1}^{-1}\left(\ddot{x}_{d}+k_{1} \dot{e}_{1}+k_{2} e_{1}-\dot{J}_{1} \dot{q}_{1}\right),
\end{gathered}
$$

where $u_{1}(t) \in \mathbb{R}^{n_{1}}$ is an auxiliary control input and $k_{1}, k_{2} \in$ $\mathbb{R}$ are positive control gains. Substituting (12) and (13) into (1) for $i=1$ and premultiplying with $M_{1}^{-1}(\cdot)$ results in

$$
\ddot{q}_{1}=J_{1}^{-1}\left(\ddot{x}_{d}+k_{1} \dot{e}_{1}+k_{2} e_{1}-\dot{J}_{1} \dot{q}_{1}\right) .
$$

Substituting the joint acceleration expression from (5) into (14) and premultiplying with $J_{1}(\cdot)$ results in the closed-loop error system

$$
\ddot{e}_{1}+k_{1} \dot{e}_{1}+k_{2} e_{1}=0_{n_{1} \times 1},
$$

from which it can be concluded that with appropriate choice of $k_{1}$ and $k_{2},\left\|e_{1}(t)\right\| \rightarrow 0$ exponentially fast.

The coordination error between the master and slave systems denoted by $e_{2}(t) \in \mathbb{R}^{n_{1}}$, is defined as

$$
e_{2} \triangleq x_{1}-x_{2} \text {. }
$$

To ensure the continuum manipulator tip tracks the master end-effector, the following feedback linearizing controller is designed for the slave system

$$
\begin{gathered}
T_{2} \triangleq M_{2} u_{2}+N_{2}-J_{2}^{T} F_{2} \\
u_{2} \triangleq J_{2}^{+}\left(\ddot{x}_{d}+k_{1} \dot{e}_{1}+k_{2} e_{1}+k_{3} \dot{e}_{2}+k_{4} e_{2}-\dot{J}_{2} \dot{q}_{2}\right)+\phi_{N}
\end{gathered}
$$

where $u_{2}(t) \in \mathbb{R}^{n_{2}}$ is an auxiliary control input, $k_{3}, k_{4} \in \mathbb{R}$ are positive control gains and $\phi_{N}(t) \in \mathbb{R}^{n_{2}}$ is a vector in the null-space of $J_{2}(\cdot)$. Substituting (17) and (18) into (1) for $i=2$, premultiplying with $M_{2}^{-1}(\cdot)$ and utilizing (15) results in

$$
\ddot{q}_{2}=J_{2}^{+}\left(\ddot{x}_{1}+k_{3} \dot{e}_{2}+k_{4} e_{2}-\dot{J}_{2} \dot{q}_{2}\right)+\phi_{N} .
$$

Substituting (6) in the right-hand-side of (19) results in

$$
\begin{aligned}
J_{2}^{+}\left(\ddot{x}_{2}-\dot{J}_{2} \dot{q}_{2}\right)= & J_{2}^{+}\left(\ddot{x}_{1}+k_{3} \dot{e}_{2}+k_{4} e_{2}-\dot{J}_{2} \dot{q}_{2}\right) \\
& +\phi_{N}-\ddot{q}_{N} .
\end{aligned}
$$

After premultiplying (20) with $J_{2}(\cdot)$ and rearranging the terms, the simplified error system is denoted as

$$
\ddot{e}_{2}+k_{3} \dot{e}_{2}+k_{4} e_{2}=0_{n_{1} \times 1} .
$$

The expression in (21) utilized the properties of the MoorePenrose pseudoinverse listed in (8), (9), and(10). Additionally

$$
J_{2} \phi_{N}=0_{n_{1} \times 1} \quad, \quad J_{2} \ddot{q}_{N}=0_{n_{1} \times 1},
$$


because $\phi_{N}(t)$ and $\ddot{q}_{N}(t)$ exist in the null space of $J_{2}(q)$.

From (21), it is clear that with an appropriate choice of $k_{3}$ and $k_{4},\left\|e_{2}(t)\right\|$ goes to zero exponentially fast.

\section{Null-Space Velocity Tracking}

In addition to the task-space tracking and coordination objectives, secondary or sub-task objectives may be required to successfully accomplish a particular task. In order to ensure the sub-task objective is achieved, an auxiliary nullspace signal $g(t) \in \mathbb{R}^{n_{2}}$, is introduced. The integration of this signal into the controller is done by designing a framework that places preferences on desirable slave configurations based on the corresponding sub-task objective. This auxiliary signal is designed to operate in the null-space of the slave continuum manipulator's Jacobian matrix $J_{2}\left(q_{2}\right)$.

As noted in [40], the null-space velocity tracking error is defined as

$$
\dot{e}_{N} \triangleq\left(I_{n_{2}}-J_{2}^{+} J_{2}\right)\left(g-\dot{q}_{2}\right)
$$

where $g(t)$ is the auxiliary null-space term, yet to be designed. The time derivative of (23) is found to be

$$
\ddot{e}_{N}=\left(I_{n_{2}}-J_{2}^{+} J_{2}\right)\left(\dot{g}-\ddot{q}_{2}\right)-J_{\phi}\left(g-\dot{q}_{2}\right)-J_{2}^{+} \dot{J}_{2} \dot{e}_{N} .
$$

Substituting (19) into (24) results in

$$
\ddot{e}_{N}=\left(I_{n_{2}}-J_{2}^{+} J_{2}\right) \dot{g}-\phi_{N}-J_{\phi}\left(g-\dot{q}_{2}\right)-J_{2}^{+} \dot{J}_{2} \dot{e}_{N}
$$

where the fact that $\phi_{N}(t)$ exists in the null-space of $J_{2}(\cdot)$ and (10) are utilized. The auxiliary function $J_{\phi}(t) \in \mathbb{R}^{n_{2} \times n_{2}}$ is based on the slave system Jacobian and defined as

$$
J_{\phi} \triangleq \dot{J}_{2}^{+} J_{2}+J_{2}^{+} \dot{J}_{2} J_{2}^{+} J_{2}
$$

The auxiliary null-space vector $\phi_{N}(t)$, introduced in (18), is designed to be

$$
\phi_{N} \triangleq\left(I_{n_{2}}-J_{2}^{+} J_{2}\right)\left(\dot{g}+k_{n} \dot{e}_{N}\right)-J_{\phi}\left(g-\dot{q}_{2}\right)
$$

where $k_{n} \in \mathbb{R}$ is a positive constant. After substituting $\phi_{N}(t)$ into (25), the expression in (27) simplifies into

$$
\ddot{e}_{N}=k_{n}\left(I_{n_{2}}-J_{2}^{+} J_{2}\right) \dot{e}_{N}-J_{2}^{+} \dot{J}_{2} \dot{e}_{N} .
$$

The auxiliary null-space vector in (27) guarantees that $\dot{e}_{N}(t) \rightarrow 0$ as $t \rightarrow \infty$ as proven in [40].

\section{Sub-Task Closed Loop Error System}

The sub-task objective will be met if a Jacobian-type nullspace matrix, $J_{s}(t) \in \mathbb{R}^{1 \times n_{2}}$ maintains full rank as shown in the condition stated in (36). To that end, when $J_{s}(t)$, yet to be defined, loses rank, the sub-task objective will not be met. To facilitate this development, an auxiliary positive function $y_{a}(t) \in \mathbb{R}$ is defined as

$$
y_{a} \triangleq \exp \left(-k_{y} \beta\left(q_{2}\right)\right)
$$

where $k_{y} \in \mathbb{R}$ is a positive constant and $\beta(\cdot) \in \mathbb{R}$ is a subtask dependent non-negative function.

The time derivative of (29) yields

$$
\dot{y}_{a}=J_{s} \dot{q}_{2}
$$

where $J_{s}(t) \in \mathbb{R}^{1 \times n_{2}}$ is defined as

$$
J_{s} \triangleq \frac{\partial y_{a}}{\partial q_{2}}
$$

Adding and subtracting the terms $J_{s} J_{2}^{+} J_{2} \dot{q}_{2}$ and $J_{s}\left(I_{n_{2}}-J_{2}^{+} J_{2}\right)\left(g-\dot{q}_{2}\right)$ respectively to the right-hand-side of (30), and substituting (4) and (23) into the expression results in

$$
\dot{y}_{a}=J_{s} J_{2}^{+} \dot{x}_{2}+J_{s}\left(I_{n_{2}}-J_{2}^{+} J_{2}\right) g-J_{s} \dot{e}_{N} .
$$

The auxiliary null-space term $g(t)$ is designed to be

$$
g=-k_{s} J_{s}^{T} y_{a}
$$

where $k_{s} \in \mathbb{R}$ is a positive constant. Substituting (33) into (32) and applying (9) results in

$$
\dot{y}_{a}=-k_{s}\left\|J_{s}\left(I_{n_{2}}-J_{2}^{+} J_{2}\right)\right\|^{2} y_{a}+J_{s} J_{2}^{+} \dot{x}_{2}-J_{s} \dot{e}_{N} .
$$

Theorem 1: The null-space term described in (33) guarantees that $y_{a}(t)$ is ultimately bounded by the inequality

$$
\left|y_{a}(t)\right| \leq \sqrt{y_{a}^{2}\left(t_{0}\right) \exp (-2 \gamma t)+\frac{\varepsilon}{\gamma}}
$$

provided that

$$
\begin{gathered}
\left\|J_{s}\left(I_{n_{2}}-J_{2}^{+} J_{2}\right)\right\|^{2}>\bar{\delta}, \\
\left\|J_{s}\left(J_{2}^{+} x_{2}-\dot{e}_{N}\right)\right\| \leq \delta_{1}, \\
k_{s}>\frac{1}{\bar{\delta} \delta_{2}},
\end{gathered}
$$

where $\varepsilon, \gamma, \bar{\delta}, \delta_{1}, \delta_{2} \in \mathbb{R}$ are positive constants.

Proof: The proof can be found in Appendix A of [41].

Remark 1: It should be noted that, the sub-task objective is met only if the sufficient conditions described by the inequalities in (36)-(38) are satisfied. Based on the analysis in Section III, the task-space tracking objective is always guaranteed and the sub-task control objective is always secondary to it. When the sub-task controller forces the end-effector of the manipulator to take a path not allowed by the task-space controller, the condition in (36) will not be satisfied; hence, (35) will not hold. Thus, careful consideration is required in the design of the desired task-space trajectory and the subtask control objective to meet the task-space tracking and sub-task objectives simultaneously.

\section{Simulation ExAmples}

A numerical simulation was performed using the MATLAB/Simulink software package to highlight the performance of the controllers proposed in (12), (17), and (23) as well as the sub-task controller $g(t)$ described in (33). The simulation was run using an aggressive sinusoidal task-space trajectory for the master system end-effector whose dynamics were computed using the standard Euler-Lagrangian model described in [43]. The link lengths for the master system were nominally chosen to be $l_{1,1}=l_{1,2}=0.6[\mathrm{~m}]$. The full dynamic model model for the Octarm is developed and described by Tatlicioglu et al. in [17] and [44] with all 
manipulator properties listed and accounted for. It should be noted that Additive White Gaussian Noise with $S N R=40$ was added into the feedback loop to simulate feedback delays or noise in sensor readings. The control gains were selected to be

$$
\begin{array}{lccc}
k_{1}=3 & k_{2}=10 & k_{3}=10 \\
k_{4}=15 & k_{y}=1 & k_{s}=1 & k_{n}=100 .
\end{array}
$$

The desired task-space trajectory, $x_{d}(t) \in \mathbb{R}^{2}$ was chosen as

$$
x_{d}=\left[\begin{array}{c}
X_{d} \\
Z_{d}
\end{array}\right]=\left[\begin{array}{c}
0+0.01 \sin (t) \\
1+0.01 \cos (t)
\end{array}\right] .
$$

This trajectory ensures that one possible configuration for the slave system is a singularity, every time the $\mathrm{x}$ coordinate of the tip crosses zero, because the manipulator sections are then completely co-linear to the z-axis, in order to test the nominal sub-task objective for the slave system of avoiding potential singular configurations, and, hence, decrease its manipulability. For this sub-task, $\beta\left(q_{2}\right)$, defined as the manipulability measure and described in [45] is chosen as

$$
\beta=\sqrt{\operatorname{det}\left[J_{2} J_{2}^{T}\right]}
$$

where $\operatorname{det}(\cdot)$ is the determinant of a matrix. It should be noted that the auxiliary signal $y_{a}(t)$ defined in (29) was chosen in order to exploit the useful properties of the exponential function. Thus, it can be seen that $0<y_{a}(t) \leq 1$ results in $\beta(\cdot) \in \mathbb{R}^{+}$.

From (41), it can be seen that $\beta\left(q_{2}\right)=0$ denotes singular configurations for the manipulator because the Jacobian $J_{2}$ will no longer be of full rank. Thus the problem is set up to ensure $\beta(\cdot)>0$, which is achieved by keeping $y_{a}(t) \leq 1$.

The continuum manipulator was initially set to be at rest with the section curvatures set near 0 (almost a singular configuration) so as to maximize $y_{a}\left(t_{0}\right)$ and demonstrate that (35) holds for this simulation. Additionally, the desired taskspace trajectory is specifically chosen to operate across tip locations consistent with the slave's singular configuration.

The controller performance is illustrated in Figures 2, and 3 , and 4. Figure 2 shows the tracking error between the desired trajectory and and the non-redundant master system as described in equation (11), while Figure 3 shows the taskspace tracking error between the non-redundant master system and the continuum slave system as described in equation (16). The ability of the controllers to avoid singularities is validated by the fact that the manipulability measure $\beta\left(q_{2}\right)$ hovers close to zero without actually reaching it as show in Figure 4. It can be seen that the slave system tracking error converges to zero much slower than the master system. While the continuum arm attempts to reach the desired tip location exponentially fast, the singularity repulsion function used for the manipulability measure $\beta\left(q_{2}\right)$ forces the manipulator to adjust its shape to an acceptable non-singular configuration.

\section{CONCLUSION}

Control of teleoperated systems with a non-redundant master and continuum slave systems was discussed. Due
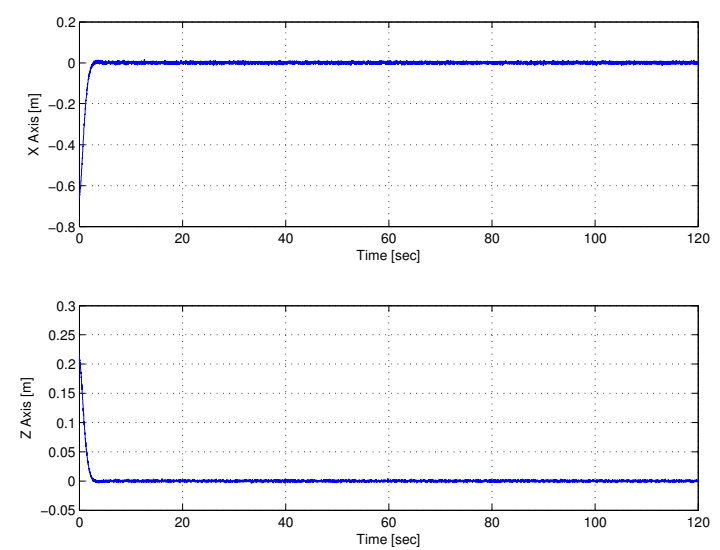

Fig. 2. Master system tracking error $e_{1}(t)$.
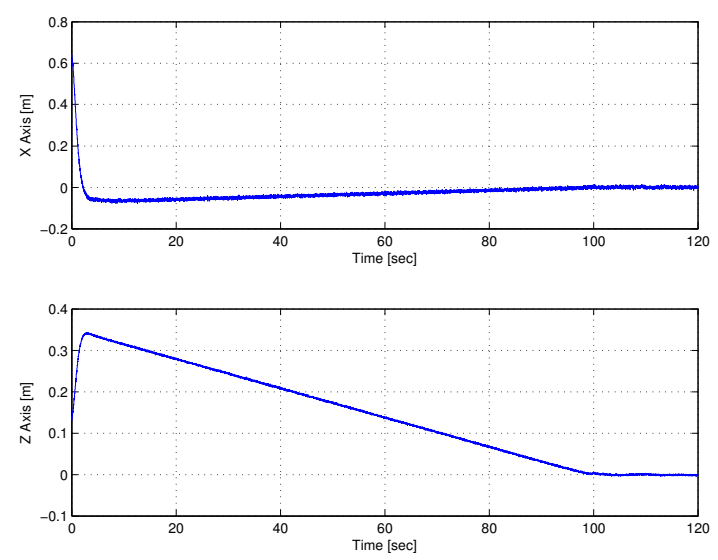

Fig. 3. Slave system tracking error $e_{2}(t)$.

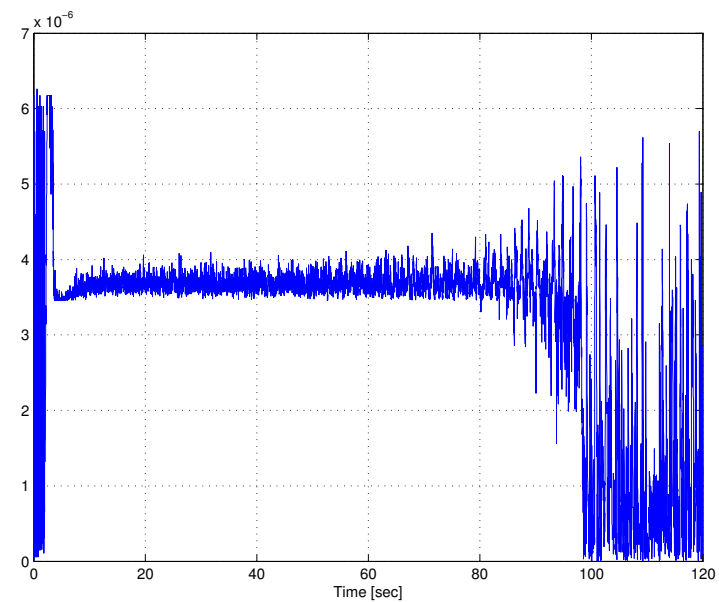

Fig. 4. Manipulability measure for the continuum arm slave system $\beta(t)$. Note that although the measure approaches zero, it never actually attains it. 
to the kinematic dissimilarities between the two systems, feedback linearizing task-space controllers are proposed, with additional sub-task terms for the slave system to allow for varied objectives in its null-space, independent of the primary master system task-space tracking objective. Numerical simulations highlight the effectiveness of the controllers along with a singularity avoidance sub-task objective for the slave system.

\section{REFERENCES}

[1] T. B. Sheridan, Telerobotics, Automation, and Human Supervisory Control. Cambridge, MA, USA: MIT Press, 1992.

[2] P. F. Hokayem and M. W. Spong, "Bilateral teleoperation: An historical survey," Automatica, vol. 42, no. 12, pp. 2035-2057, 2006.

[3] G. Robinson and J. Davies, "Continuum robots - a state of the art," in Proc. IEEE Int. Conf. Robot. Autom., Detroit, MI, 1999, pp. 2849 2854.

[4] R. Buckingham and A. Graham, "Snaking around in a nuclear jungle," Industrial Robot: An Int. Jour, vol. 32, no. 2, pp. 120-127, 2005.

[5] G. Immega and K. Antonelli, "The ksi tentacle manipulator," in Proc. IEEE Int. Conf. Robot. Autom., Nagoya, Japan, 1995, pp. 3149-3154.

[6] D. Lane, B. Davies, G. Robinson, D. O'Brien, J. Sneddon, E. Seaton, and A. Elfstrom, "Practical kinematics for real-time implementation of continuum robots," IEEE Jour. Ocean. Eng., vol. 24, no. 1, pp. 96-111, Jan. 1999.

[7] H. Ohno and S. Hirose, "Design of slim slime robot and its gait of locomotion," in Proc. IEEE/RSJ Int. Conf. Intel. Robot. Syst., Maui, HI, 2001, pp. 707-715.

[8] S. Hirose, Biologically Inspired Robots. New York, NY: Oxford University Press, 1993.

[9] I. Walker, D. Dawson, T. Flash, F. Grasso, R. Hanlon, B. Hochner, W. Kier, C. Pagano, C. Rahn, and Q. Zhang, "Continuum robot arms inspired by cephalopods," in Proc. SPIE Conf. Unmanned Ground Veh. Tech., Orlando, FL, 2005, pp. 303-314.

[10] K. Suzimori, S. Iikura, and H. Tanaka, "Development of a flexible microactuator and its applications to robotic mechanisms," in Proc. IEEE Int. Conf. Robot. Autom., Sacramento, CA, 1991, pp. 16221627

[11] D. Trivedi, C. Rahn, W. Kier, and I. Walker, "Soft robotics: Biological inspiration, state of the art, and future research," Applied Bionics and Biomech., vol. 5, no. 2, pp. 99-117, 2008.

[12] R. W. III and B. Jones, "Design and modeling of constant curvature continuum robots: A review," Int. Jour. Robot. Res., vol. 29, no. 13, pp. 1661-1683, 2010.

[13] G. Chirikjian, "Hyper-redundant manipulator dynamics: A continuum approximation," Advanced Robotics, vol. 9, no. 3, pp. 217-243, 1995.

[14] W. Khalil, G. Gallot, O. Ibrahim, and F. Boyer, "Dynamic modeling of a 3-d serial eel-like robot," in Proc. IEEE Int. Conf. Robot. Autom., Barcelona, Spain, 2005, pp. 1282-1287.

[15] F. Matsuno and H. Sato, "Trajectory tracking control of snake robots based on dynamic model," in Proc. IEEE Int. Conf. Robot. Autom., Barcelona, Spain, 2005, pp. 3040-3045.

[16] H. Mochiyama and T. Suzuki, "Dynamics modelling of a hyperflexible manipulator," in Proc. of the SICE Ann. Conf., Osaka, Japan, 2002, pp. 1505-1510.

[17] E. Tatlicioglu, I. D. Walker, and D. M. Dawson, "Dynamic modeling for planar extensible continuum robot manipulators," Int. J. of Rob. and Autom., vol. 24, no. 4, pp. 356-366, 2009.

[18] M. Ivanescu and V. Stoian, "A variable structure controller for a tentacle manipulator," in Proc. IEEE Int. Conf. Robot. Autom., Nagoya, Japan, 1995, pp. 3155-3160.

[19] _ - "A controller for hyper-redundant cooperative robots," in Proc. IEEE/RSJ Int. Conf. Intel. Robot. Syst., Vancouver, Canada, 1998, pp. $167-172$.

[20] M. Ivanescu, N. Popescu, and D. Popescu, "A variable length tentacle manipulator control system," in Proc. IEEE Int. Conf. Robot. Autom., Barcelona, Spain, 2005, pp. 3274-3279.

[21] A. Kapadia, I. Walker, D. Dawson, and E. Tatlicioglu, "A new approach to extensible continuum robot control using the slidingmode," Intl. J. Comp. Tech. and App., vol. 2, no. 4, pp. 293-300, 2011
[22] A. Kapadia and I. Walker, "Task space control of extensible continuum manipulators," in Proc. IEEE/RSJ Int. Conf. Intel. Robot. Syst., San Francisco, Ca, 2011, pp. 1087-1092.

[23] M. Csencsits, B. Jones, W. McMahan, V. Iyengar, and I. Walker, "User interfaces for continuum robot arms," in Proc. IEEE/RSJ Int. Conf. Intel. Robot. Syst., Edmonton, Canada, 2005, pp. 3011-3018.

[24] K. Kim, W. K. Chung, and I. H. Suh, "Accurate force reflection for kinematically dissimilar bilateral teleoperation systems using instantaneous restriction space," in Proc. IEEE Int. Conf. Robot. Autom., Orlando, FL, 2006, pp. 3257-3262.

[25] J. Herndon, W. Hamel, and D. Kuban, "Traction-drive telerobot for space manipulation," in Proc. IEEE Int. Conf. Robot. Autom., Raleigh, NC, 1987, pp. 450-455.

[26] C. C. Nguyen, Z.-L. Zhou, and G. E. Mosier, "Joint-space adaptive control of a redundant telerobot manipulator," in Proc. of Int. Symp. on Intel. Cont., Albany, NY, 1989, pp. 59-65.

[27] J. F. Jansen, R. L. Kress, S. M. Babcock, and W. R. Hamel, "Stiffness control of teleoperators with redundant, dissimilar kinematics," in Proc. of Int. Conf. on System Engineering, Pittsburgh, PA, 1990, pp. 97-100.

[28] J. F. Jansen and R. L. Kress, "Control of a teleoperator system with redundancy based on passivity condition," in Proc. IEEE Int. Conf. Robot. Autom., Sacramento, CA, 1991, pp. 478-484.

[29] D.-Y. Hwang and B. Hannaford, "Teleoperation performance with a kinematically redundant slave robot," Int. J. Robot. Res., vol. 17, no. 6 , pp. 579-597, 1998.

[30] D. P. T. Nanayakkara, K. Kiguchi, T. Murakami, K. Watanabe, and K. Izumi, "Skillful adaptation of a 7-dof manipulator to avoid moving obstacles in a teleoperated force control task," in Proc. of IEEE Int. Symposium on Indust. Electronics.

[31] M. Goel, A. A. Maciejewski, V. Balakrishnan, and R. W. Proctor, "Failure tolerant teleoperation of a kinematically redundant manipulator: An experimental study," IEEE Trans. Syst., Man, Cybern. A, vol. 33, no. 6, pp. 758-765, 2003.

[32] B. Stanczyk and M. Buss, "Development of a telerobotic system for exploration of hazardous environments," in Proc. IEEE/RSJ Int. Conf. Intel. Robot. Syst., Sendai, Japan, 2004, pp. 2532-2537.

[33] M. Ueberle, N. Mock, and M. Buss, "Vishard10, a novel hyperredundant haptic interface," in Proc. IEEE Symp. Haptic Interf. for Virt. Envir. \& Teleop. Sys., Chicago, IL, 2004, pp. 58-65.

[34] A. Peer, B. Stanczyk, and M. Buss, "Haptic telemanipulation with dissimilar kinematics," in Proc. IEEE/RSJ Int. Conf. Intel. Robot. Syst. Alberta, Canada, 2005, pp. 3493-3498.

[35] Y. Komoguchi, A. Peer, and M. Buss, "Control and performance evaluation of a new redundant haptic interface," in Proc. of the SICE Ann. Conf., Kagawa, Japan, 2007, pp. 2671-2676.

[36] M. Hayakawa, K. Hara, D. Sato, A. Konno, and M. Uchiyama, "Singularity avoidance by inputting angular velocity to a redundant axis during cooperative control of a teleoperated dual-arm robot," in Proc. IEEE Int. Conf. Robot. Autom.

[37] F. Gosselin, C. Andriot, F. Bergez, and X. Merlhiot, "Widening 6-dof haptic devices workspace with an additional degree of freedom," in Proc. 2nd Joint EuroHaptics Conf. and Symp. Haptic Interf. for Virt. Env. \& Teleop. Sys., Tsukuba, Japan, 2007, pp. 452-457.

[38] N. Nath, E. Tatlicioglu, and D. M. Dawson, "Teleoperation with kinematically redundant robot manipulators with sub-task objectives,' Robotica, vol. 27, no. 7, pp. 1027-1038, 2009.

[39] H. Das, T. B. Sheridan, and J.-J. E. Slotine, "Kinematic control and visual display of redundant teleoperators," in Proc. of Int. Conf. on Systems, Man and Cybernetics, Cambridge, MA, 1989, pp. 1072-1077.

[40] P. Hsu, J. Hauser, and S. Sastry, "Dynamic control of redundant manipulators," J. of Robotic Systems, vol. 6, no. 3, pp. 133-148, 1989.

[41] E. Tatlicioglu, Control of Nonlinear Mechatronic Systems. Germany: VDM Verlag Dr. Mueller e.K., 2008.

[42] F. L. Lewis, D. M. Dawson, and C. T. Abdallah, Robot Manipulator Control: Theory and Practice. New York, NY: Marcel Dekker, 2004

[43] M. W. Spong and M. Vidyasagar, Robot Dynamics and Control. New York, NY, USA: John Wiley and Sons, Inc., 1989.

[44] E. Tatlicioglu, I. D. Walker, and D. M. Dawson, "New dynamic models for planar extensible continuum robot manipulators," in Proc. IEEE/RSJ Int. Conf. Intel. Robot. Syst., San Diego, CA, USA, 2007, pp. $1485-1490$.

[45] L. Sciavicco and B. Sicilano, Modeling and Control of Robot Manipulators. New York, NY, USA: McGraw-Hill Co., 1996. 